\title{
A note on the spreading rate and virtual origin of a plane turbulent jet
}

\author{
By NIKOLAS E. KOTSOVINOS
}

W. M. Keck Laboratory of Hydraulics and Water Resources, California Institute of Technology, Pasadenat

(Received. 24 November 1975)

The reported experimental coefficients describing the spreading rate and virtual origin of a plane turbulent jet exhibit substantial scatter. The hypothesis is made that the basic reason for these variations is that the growth of the jet is not linear on a large scale. Existing experimental results are used to support this hypothesis.

\section{Introduction}

The spreading rate and the virtual origin of a turbulent plane jet issuing through a slot of thickness $D$ and length $W$ into a still ambient fluid are examined. A Cartesian system of co-ordinates $O x y$ is used where the $x$ axis coincides with the jet axis.

The plane turbulent jet has been the object of experimental investigations for many years (see table 1). Normally an experimenter measures the lateral distribution of the mean axial velocity $\bar{u}(x, y)$ at several stations at various distances $x$ from the jet orifice and calculates the half-width $b(x)$ from the relation

$$
\bar{u}(x, \pm b(x))=\frac{1}{2} \bar{u}(x, 0) \text {. }
$$

The half-widths $b(x)$, normalized by the slot thickness $D$, are plotted versus the non-dimensional distance from the jet orifice $x / D$, and a straight line of the form

$$
b(x) / D=K_{1}\left(x / D+K_{2}\right)
$$

is fitted to the data points. The coefficient $K_{1}$ is a measure of the spreading rate of the jet. The virtual origin of the jet is obtained from the relation

$$
x_{0}=-K_{2} D \text {. }
$$

The coefficients $K_{1}$ and $K_{2}$ derived from published data are tabulated in table 1 , where $l_{\min }$ is the distance of the closest measurement from the jet exit and $l_{\max }$ is the distance of the furthest measurement from the jet exit. The parameters $W / D$ (aspect ratio of the slot) and $l_{\max } / W$ determine how closely the jet approximates two-dimensional flow in the region of measurements. The experimental results of van der Hegge Zijnen (1957) and Heskestad (1965) indicate that jets with $W / D$ greater than 10 to 20 and $l_{\max } / W$ less than two approximate the two-dimensional case in the plane of symmetry.

An examination of table 1 reveals that the coefficients $K_{1}$ and $K_{2}$ vary substantially from investigator to investigator. Some investigators find the location

$\dagger$ Present address: Sourmeli 5, Athens 108, Greece. 


\begin{tabular}{|c|c|c|c|c|c|c|c|}
\hline Experimenter(s) & $\begin{array}{c}\text { Slot } \\
\text { aspect } \\
\text { ratio }\end{array}$ & $l_{\min } \mid D$ & $l_{\max } / D$ & $l_{\max } / W$ & $\begin{array}{c}R e \\
\times 10^{-3}\end{array}$ & $K_{1}$ & $K_{2}$ \\
\hline Albertson et al. (1950) & $20-5760$ & 24 & 2300 & $1 \cdot 20$ & 10 & $0 \cdot 128$ & - \\
\hline Flora \& Goldschmidt (1969) & 50 & - & 90 & $1 \cdot 80$ & 20 & $0 \cdot 11$ & $0 \cdot 0$ \\
\hline Förthmann (1934) & 21 & 0 & 25 & $1 \cdot 19$ & 70 & 0.096 & $0 \cdot 60$ \\
\hline Goldschmidt \& Eskinazi (1966) & 16 & 15 & 67 & $4 \cdot 20$ & 16 & $0 \cdot 099$ & $-0 \cdot 66$ \\
\hline Heskestad (1965) & 120 & 47 & 155 & $1 \cdot 30$ & 35 & $0 \cdot 11$ & $-4 \cdot 2$ \\
\hline Jenkins \& Goldschmidt (1973) & 24 & 30 & 60 & $2 \cdot 5$ & 14 & $0 \cdot 088$ & $4 \cdot 5$ \\
\hline Kotsovinos (1975) & 54 & 20 & 94 & $1 \cdot 45$ & 2 & $0 \cdot 109$ & $-2 \cdot 5$ \\
\hline & 13 & 14 & 37 & $2 \cdot 85$ & $\mathbf{2}$ & 0.087 & $6 \cdot 5$ \\
\hline Mih \& Hoopes (1972) & 59 & 一 & $\mathbf{3} 00$ & $5 \cdot 08$ & 20 & $0 \cdot 117$ & 一 \\
\hline Miller \& Comings (1957) & 40 & 0 & 40 & $1 \cdot 0$ & 20 & 0.096 & $1 \cdot 6$ \\
\hline Nakaguchi (1961) & 133 & - & 100 & 0.75 & 10 & $0 \cdot 106$ & $0 \cdot 3$ \\
\hline
\end{tabular}

of the virtual origin to be upstream from the jet orifice and others find it to be downstream. The spreading rates $K_{1}$ found by Jenkins \& Goldschmidt (1973) and by Albertson et al. (1950) differ by about 35\%. Newman (1965) attributed these variations to internal boundary layers at the slot (different nozzle shapes) and to low aspect ratio. However, Flora \& Goldschmidt (1969) performed an experimental investigation of the plane jet using nine different nozzle shapes (abrupt, gradual, circular, etc.) in an attempt to correlate the nozzle shape with variations in $K_{1}$ and $K_{2}$. They reported that changes in the contracting section did not influence the location of the virtual origin nor the spreading rate of the jet, neither did they reveal any pattern in the scatter of the experimental results of earlier investigators.

As can readily be seen from table 1 , there seems to be no correlation between the aspect ratio and the spreading rate or the location of the virtual origin. There has been no systematic investigation of the effect of the initial jet turbulence levels.

Hence very little appears to be known about any possible pattern to the location of the virtual origin or the spreading rate. In this paper, a hypothesis is made about the origin of these variations, and is compared with existing experimental data.

\section{Analysis and test of a hypothesis}

The hypothesis made in this paper is that the variations in $K_{1}$ and $K_{2}$ are most likely due to the fact that the spreading rate is not precisely linear on a large scale. It is further bypothesized that the true growth of the velocity half-width has the shape of the continuous line in figure 1. Given such a curve experimenters $A$ and $B$ would fit straight lines to their experimental results, thus indicating linear growth. Possibly experimenter $A$ could not extend his region of measurements to a greater $x / D$, perhaps because of restrictions on the aspect ratio of the slot and the requirement of two-dimensionality. Experimenter $B$ possibly could not extend his region of measurements closer to the origin because the physical 


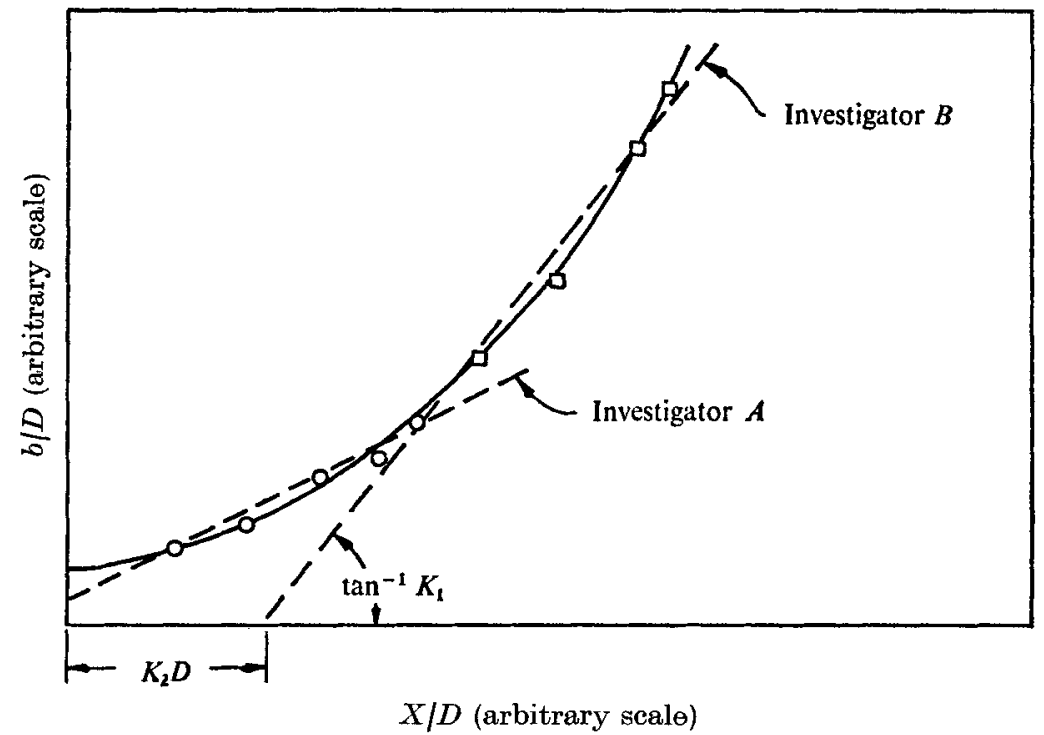

Figure 1. Hypothesis concerning the nonlinear spread of the mean velocity profile of a turbulent jet. $\bigcirc$, investigator $A ; \square$, investigator $B$.

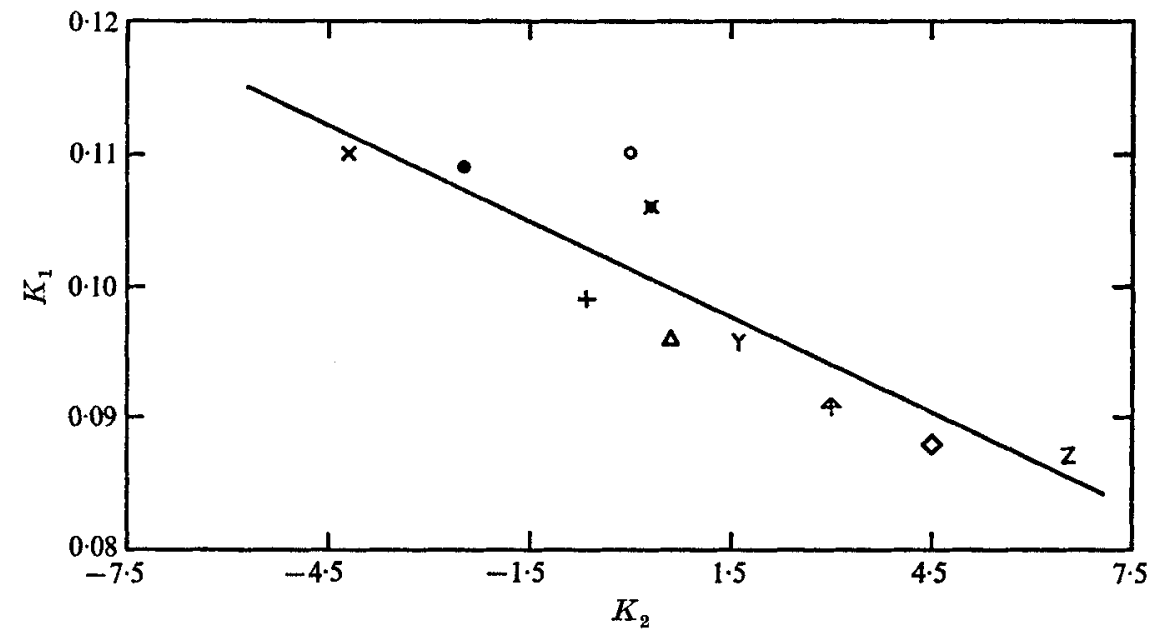

FIGURE 2. Correlation of spreading rate $K_{1}$ and non-dimensional virtual origin $K_{2}$. $\bigcirc$, Flora \& Goldschmidt; $\triangle$, Förthmann; +, Goldschmidt; $\times$, Heskesad; $\diamond, 4$, Jenkins \& Goldschmidt;,$Z$, Kotsovinos; Y, Miller; $\&$, Nakaguchi.

dimensions of the jet profile (say in $\mathrm{mm}$ ) would be too small to ensure accurate experimental results. Obviously, experimenter $A$ finds a smaller slope (i.e. a smaller coefficient $K_{1}$ ) and a negative virtual origin whereas experimenter $B$ finds a positive virtual origin and a greater $K_{1}$.

The preceding discussion indicates that, if this hypothesis is correct, a correlation should be found between the experimental results (namely $K_{1}, K_{2}$ and $l_{\max } / D$ ) of the different experimenters.

The coefficients $K_{1}$ from each jet investigator are plotted versus the coefficient 


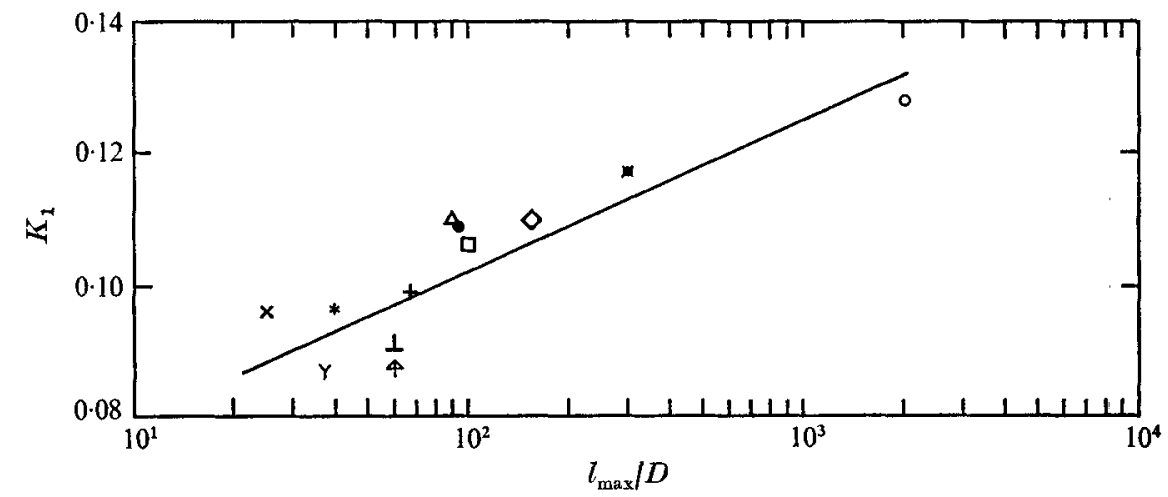

FiguRE 3. Spreading rate $K_{1}$ as a function of non-dimensional distance $l_{\mathrm{max}}[D . \bigcirc$, Albertson et al.; $\triangle$, Flora \& Goldschmidt; $\times$, Förthmann; +, Goldschmidt; $\diamond$, Heskestad; 4,1 , Jenkins \& Goldschmidt; $\bullet, Y$, Kotsovinos; Mih \& Hoopes; *, Miller \& Comings; $\square$, Nakaguchi.

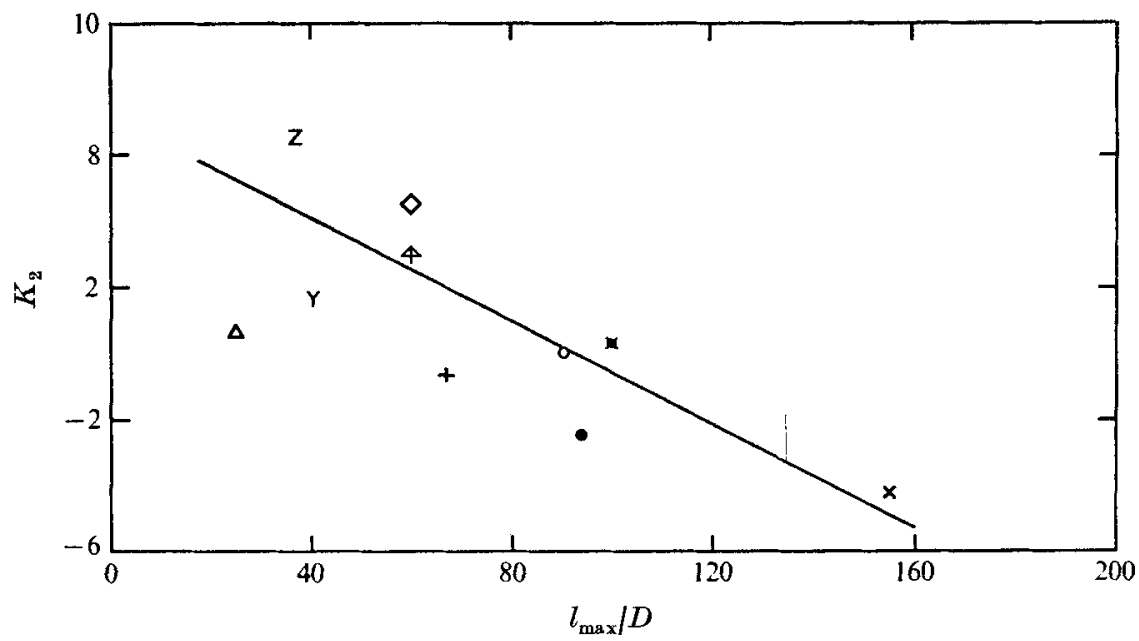

FIGURE 4. Non-dimensional virtual origin $K_{2}$ as a function of non-dimensional distance $l_{\max } / D$. Symbols as in figure 2.

$K_{2}$ in figure 2. The coefficient $K_{1}$ is plotted versus $l_{\max } / D$ in figure 3 , and the coefficient $K_{2}$ is plotted versus $l_{\max } / D$ in figure 4 . It is interesting to note (figure 2 ) that the coefficients $K_{1}$ and $K_{2}$ do not vary randomly from investigator to investigator, but vary as predicted by the hypothesis, i.e. when $K_{1}$ increases, $K_{2}$ decreases and becomes negative, giving a positive virtual origin $x_{0}=-K_{2} D$. Also the coefficient $K_{1}$ increases as $l_{\max } / D$ increases and $K_{2}$ decreases as $l_{\max } / D$ increases, as predicted. The coefficients $K_{1}$ and $K_{2}$ given by any experimenter should therefore apparently be viewed as the best linear approximation, for each experimenter, of the growth of the half-width in the region $l_{\min } / D<x / D<l_{\max } / D$. Equation (2) with coefficients so determined should not be extrapolated outside this region.

Keeping in mind the above ideas, the jet half-width $b(x) / D$ is plotted versus the distance $x / D$ in figure 5 (where $x$ varies from a few orifice widths to approximately 200 orifice widths) and in figure 6 (where $x$ varies from a few to 2300 orifice widths). 


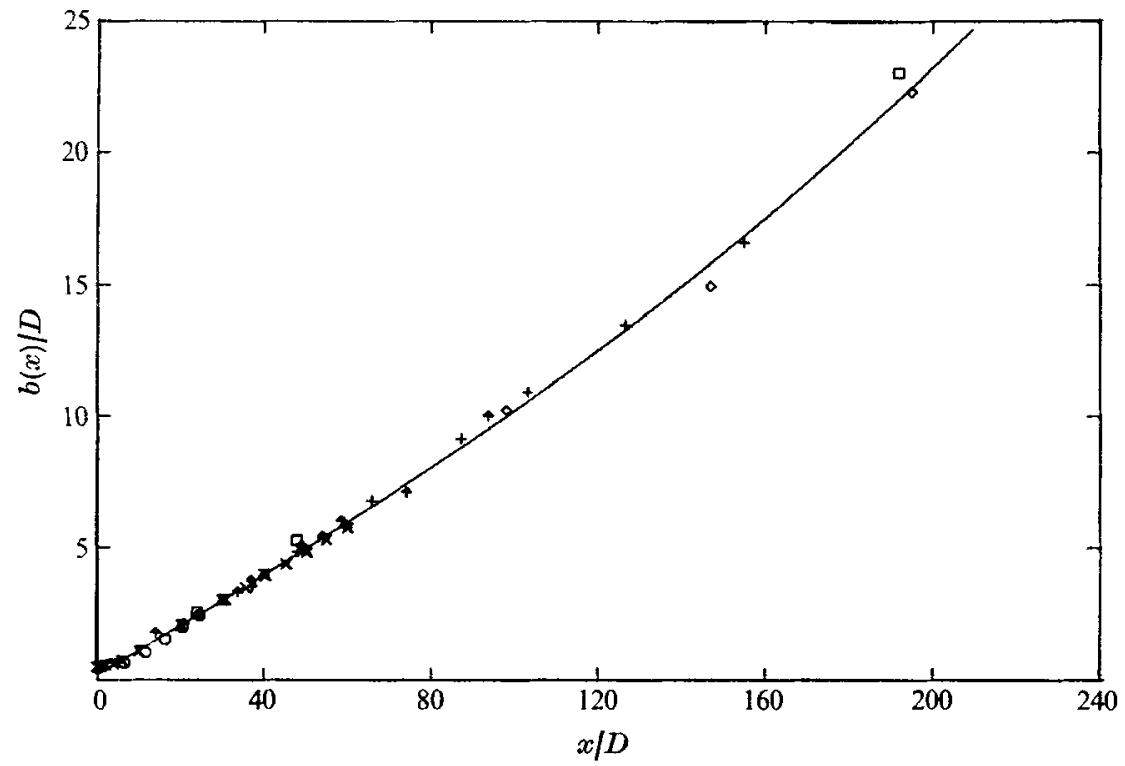

Figure 5. Nonlinear spreading of half-width of a plane jet for $x<200 D$. ___, equation (4); $\square$, Albertson et al.; $\bigcirc$, Förthmann; $\triangle$, Goldschmidt \& Eskinazi; +, Heskestad; $\times$, Jenkins \& Goldschmidt; 4 , Kotsovinos; $\diamond$, Knystautas;, , Miller \& Comings.

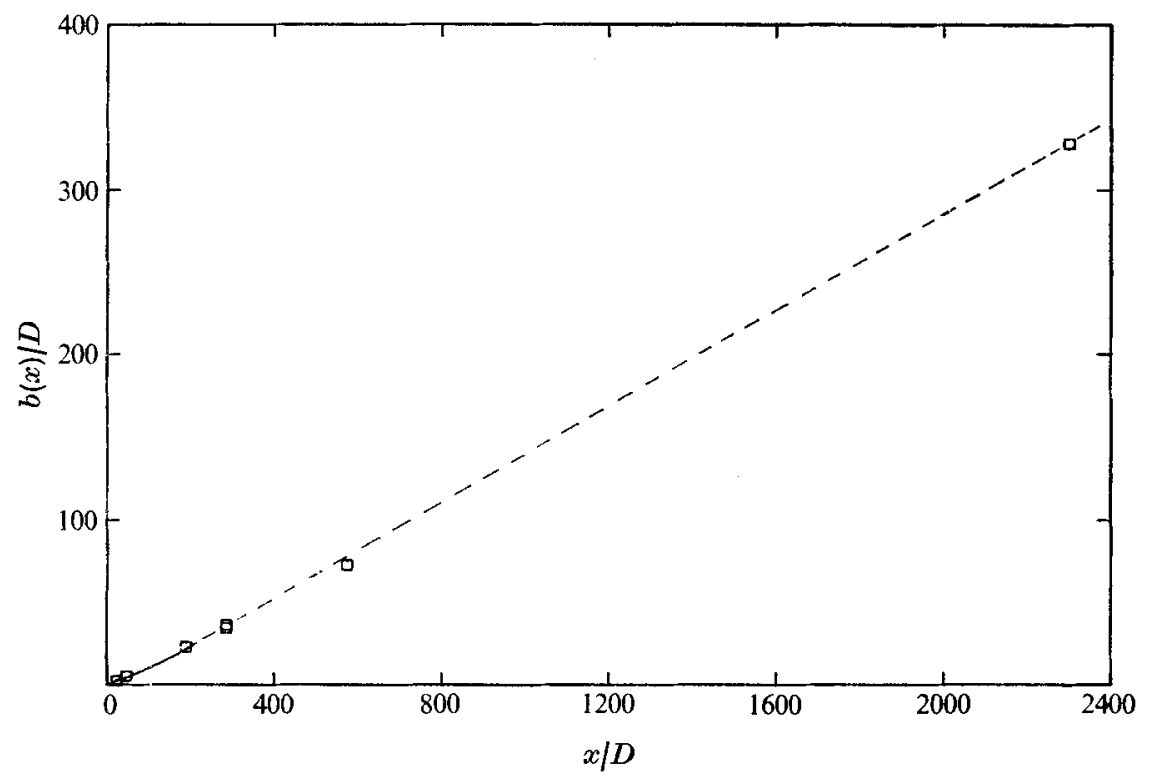

Figure 6. Spread of half-width for $6 D<x<2300 D$. _- equation (4); ,$-- d b / d x \approx 0 \cdot 14 ; \square$, Albertson et al. 
The curve which has been fitted (in the least-squares sense) in figure $\tilde{5}$ is the third-order polynomial

$$
b(x) / D=0 \cdot 228+0 \cdot 0913(x / D)+0 \cdot 00005101(x / D)^{2}+0 \cdot 000000331(x / D)^{3}
$$

and of course is valid only in a region extending up to 200 diameters. It is apparent from figure 5 that the jet growth is unique. The reported variations in the spreading rate $K_{1}$ and the virtual origin are due not to variations in the jet structure, but to the fact that the jet half-width $b(x)$ is a 'weak' nonlinear function of $x$. The jet spreading rate $K_{1}$, defined as $d b(x) / d x$, is an increasing function of $x$ for $x$ up to approximately 300 orifice widths. From 300 to 2300 orifice widths a straight line with slope 0.14 fits closely the experimental results of Albertson et al. (1950), but more experimental data are really needed in the region extending from 200 to several thousand orifice widths downstream to determine accurately how the jet grows in this region and to discover if an asymptotic linear growth exists. However, at distances of several thousand orifice widths from the jet exit, the power of the jet (flux of energy) may decrease to such levels that relaminarization of the turbulent jet may partially occur, which may make the search for an asymptotic growth unrealistic.

The true position of the virtual origin is clearly upstream of the jet orifice. It can be determined from $(4)$ that $b\left(x_{0}\right)=0$ at $x_{0} \approx-2 \cdot 5 D$, i.e. $K_{2} \approx 2 \cdot 5$.

\section{Conclusion}

The growth of the half-width of the plane turbulent jet is not exactly linear on a large scale. The reported variations in the coefficients describing the spreading rate and the virtual origin arise mainly from this nonlinear growth. An explanation for this nonlinear growth is not immediately apparent.

The author is grateful to Professor E.J.List for his perspicacious and enlightening comments. Financial assistance provided by the National Science Foundation under Grant ENG 75-02985 is greatly appreciated.

\section{REFERENCES}

Albertson, M. L., Dat, Y. B., Jensen, R. A. \& Rouse, H. 1950 Diffusion of submerged jets. Trans. A.S.C.E. 115, 639-664.

Flora, J. \& Goldschmidt, V. 1969 Virtual origins of a free plane turbulent jet. A.I.A.A. J. 7, 2344-2346.

Förthmann, E. 1934 Über turbulente Strahlausbreitung. Ing. Arch. 5, 42-54. (English trans. 1936 N.A.C.A. Tech. Memo. no. 789.)

Gordschmid, V. 1964 Two phase flow in a two-dimensional turbulent jet. Ph.D. thesis, Syracuse University.

Goldschmidt, V. \& Eskinazi, S. 1966 Two phase turbulent flow in a plane jet. J. Appl. Mech. 33, 735-747.

HegGe ZiJnen, B. G. van Der 1957 Measurements of the velocity distribution in a plane turbulent jet of air. Appl. Sci. Res. 7, 256-276.

Heskestad, G. 1965 Hot wire measurements in a plane turbulent jet. J. Appl. Mech. 32, 721-734 (erratum, Sept. 1966, p. 710). 
Jenkins, P. E. \& Goldschmidt, V. 1973 Mean temperature and velocity in a plane turbulent jet. Trans. A.S.M.E., J. Fluids Engng, 95, 581-584.

Knystautas, R. 1964 The turbulent jet from a series of holes in line. Aero. Quart. 15, 1-28.

Kotsovinos, N. E. 1975 A study of the entrainment and turbulence in a plane turbulent jet. W. M. Keck Lab. Hyd. Water Res., California Inst. Tech., Rep. KH-R-32.

Mrr, W. C. \& Hoopes, J. A. 1972 Mean and turbulent velocities for plane jet. J. Hyd. Div. A.S.C.E. 98, 1274-1294.

Miller, D. \& Cominas, E. 1957 Static pressure distribution in the free turbulent jet. J. Fluid Mech. 3, 1-16.

NakagUChi, H. 1961 Jet along a curved wall. University of Tokyo Res. Memo. no. 4 (as quoted by Newman 1965).

Newman, B. 1965 Turbulent jets and wakes in a pressure gradient. Symp. Fluid Mech. Internal Flow, Warren, Michigan (ed. G. Sovran). 